\title{
Study of I 38 Neisseria meningitidis strains isolated from blood or cerebrospinal fluid in Lombardy between 2007 and 2010
}

\author{
Laura Daprai', Maria Laura Garlaschi', Giuseppina Scelza', Damiano Picicco', \\ Maria Cristina Garlaschi', Riccardo Rubini', Gloria Defilippi', Anna Pavan², \\ Maria Gramegna ${ }^{3}$, Erminio Torresani' \\ I Laboratorio Centrale Analisi Chimico Cliniche e Microbiologia Fondazione IRCCS Ca' Granda Ospedale Maggiore \\ Policlinico, Milano \\ 2 Direzione Sanitaria Fondazione IRCCS Ca' Granda Ospedale Maggiore Policlinico, Milano \\ 3 Direzione generale Sanità, Regione Lombardia. UO: Governo della Prevenzione e Tutela Sanitaria, Milano
}

Key words: Neisseria meningitidis, Serotyping, Genotyping

Studio di 138 ceppi di Neisseria meningitidis isolati da sangue e/o da liquido cefalo-rachidiano in Lombardia nel periodo 2007-2010

\section{SUMMARY}

Neisseria meningitidis, Streptococcus pneumoniae, and Haemophilus influenzae type b cause the majority of cases of bacterial septicaemia in children and young adults. Disease epidemiology is evolving rapidly due to the introduction of vaccines and changing in bacterial antibiotic-resistance patterns. (Asymptomatic nasopharyngeal colonization with Neisseria meningitides occurs in $5-10 \%$ of adult).

The aim of this study was to calculate the frequency of each serogroup of this pathogens involved in invasive infection and to study susceptibility to antibiotics of these strains.

Between March 2007 and June 2010 we received, from 43 hospitals of Lombardy, 138 strains of Neisseria meningitidis, from 138 patients aged (2-80yrs).

The most frequent serogroup was B (58\%), followed by serogroup C (34\%), serogroup G (4\%) and W I 35 (2\%). Serogroup $A$ end $X$ accounted for $1 \%$ of invasive infection, each.

We observed a decrease in susceptibility towards penicillin in $38 \%$ of strains.

In addition we studied, by REP- PCR, genotype of 9 strains selected on the basis of epidemiological data. Among these strains, 3 different clusters according to the 3 small epidemic outbreaks occurred between June and September 2009, were recognised. Seven of these strains, although belonged to the same serogroup, brought about two different clusters.

The present findings demonstrated that phenotypic data are not sufficient to define epidemic clusters, therefore molecular genotyping is required.

\section{INTRODUZIONE}

Streptococcus pneumoniae, Neisseria meningitidis (NM) e Haemophilus influenzae type b sono una delle principali cause di meningite batterica e setticemia in bambini ed in giovani adulti, l'uomo è l'unico ospite e habitat $(1,3)$.

Il meningococco è un diplococco Gram-negativo che risiede nell'epitelio naso-faringeo, colonizza il 5-10\% della popolazione adulta nella sua forma non patogena (4).

Il fenotipo di Neisseria meningitidis è dato dal sierogruppo definito in base alle caratteristiche antigeniche degli antigeni capsulari (A, B, C, D, X, Y, Z, 29E, W135, H, T, K, L), dal sierotipo definito in base alle OMPs di classe 2 e 3 e dal sierosottotipo in base alle OMP di classe $1(10,11)$.
L'epidemiologia della malattia negli ultimi anni sta cambiando rapidamente in seguito all'introduzione delle pratiche vaccinali (9) e all'insorgenza di nuove resistenze batteriche $(2,8,12)$.

Dal 2007 la Regione Lombardia ha individuato il nostro Istituto come Centro di Riferimento per la sorveglianza delle patologie invasive da $S$. pneumoniae e da $N$. menigitidis.

Lo scopo dello studio è quello di definire, sui ceppi isolati da pazienti con malattia invasiva, $\mathrm{i}$ sierogruppi più frequenti, la sensibilità agli antibiotici e l'individuazione dei piccoli cluster epidemici attraverso la genotipizzazione.

\section{MATERIALI E METODI}

Nel periodo compreso fra Marzo 2007 e Giugno

\section{Corresponding author: Laura Garlaschi}

Laboratorio Centrale Analisi Chimico Cliniche e Microbiologia - Fondazione IRCCS "Ca' Granda” Ospedale Maggiore Policlinico - Padiglione Devoto;Via San Barnaba, 8 - Milano - Tel.: 02 55032485; Fax: 3476767962

E-mail: Igarlaschi@policlinico.mi.it 
2010 sono pervenuti al nostro centro 138 ceppi di $\mathrm{NM}$, isolati da altrettanti pazienti di età compresa fra 2-80aa provenienti da 43 ospedali lombardi. Il sierogruppo è stato eseguito con antisieri Remel. La sensibilità agli antibiotici, è stata eseguita mediante E-Test (bioMérieux), e interpretata secondo i criteri EUCAST. Il sierogruppo di 20 ceppi è stato confermato mediante tipizzazione molecolare effettuata con metodica Real-Time PCR Neisseria meningitidis/Serogroup A, B, C, $\mathrm{W} 135$ \& Y (Diagenode). Il genotipo è stato determinato su 9 ceppi di NM, mediante tecnica di repPCR (Repetitive-Sequence-Based-PCR) utilizzando il Sistema DiversiLab (bioMérieux). La scelta dei ceppi è stata determinata dai dati epidemiologici dei pazienti.

\section{RISULTATI}

La distribuzione delle diverse classi di età è riportata nella Figura I.

Il $58 \%$ dei ceppi è risultato di gruppo $\mathrm{B}$, il $34 \%$ di gruppo C, il 4\% di gruppo Y, il $2 \%$ di gruppo W135, il gruppo A e X rappresentano 1'1\% dei ceppi (Figura II). La distribuzione dei sierogruppi nelle diverse fasce di età è riportata nella Figura III.

La MIC50 e la MIC90 $(\mu \mathrm{g} / \mathrm{ml})$ sono rispettivamente per penicillina 0.047 e 0.25 (38\% di diminuita sensibilità), per rifampicina, 0.01 e 0.094 ( $2 \%$ di resistenza), per ciprofloxacina 0.004 e $0.012(<1 \%$ di resistenza), per ceftriaxone $<0.002$ e $0.003(0 \%$ di resistenza) come si può vedere nella Figura IV.

Nel periodo compreso fra giugno e settembre 2009, si sono verificate 9 meningiti batteriche da $\mathrm{NM}$ che, in funzione dei dati epidemiologici dei pazienti si possono riferire a 3 piccole epidemie. L'analisi molecolare eseguita sui 9 ceppi di NM isolati dal liquor di questi pazienti mediante una tecnica di Rep PCR, ha messo in evidenza 3 cluster diversi, riferibili alle 3 epidemie.

Il primo cluster era composto da 4 ceppi (range di similarità 97.8\%-99.4\%), il secondo da 3 ceppi (range di similarità $98.4 \%-99 \%$ ), il terzo da 2 ceppi con similarità del 99.4\% (Figura V).

L'analisi molecolare eseguita sui 9 ceppi di NM, isolati dal liquor di questi pazienti mediante una tecnica di Rep PCR ha messo in evidenza $3 \mathrm{clu}$ ster diversi, riferibili a tre epidemie.

Il pattern 1 (sierogruppo C, fenotipo C:2a:P1.5) è costituito dai ceppi da 1 a 4 che sono indistinguibili tra loro (Figura VI). Il pattern 2 (sierogruppo C, fenotipo C:2a:P1.5) comprende i ceppi da 5 a 7 e si distingue dal precedente per un picco caratteristico a circa 650 datapoints ed una spalla a circa 750 datapoints. Nonostante sia presente una elevata similarità fra i primi 2 pattern, questi posso-

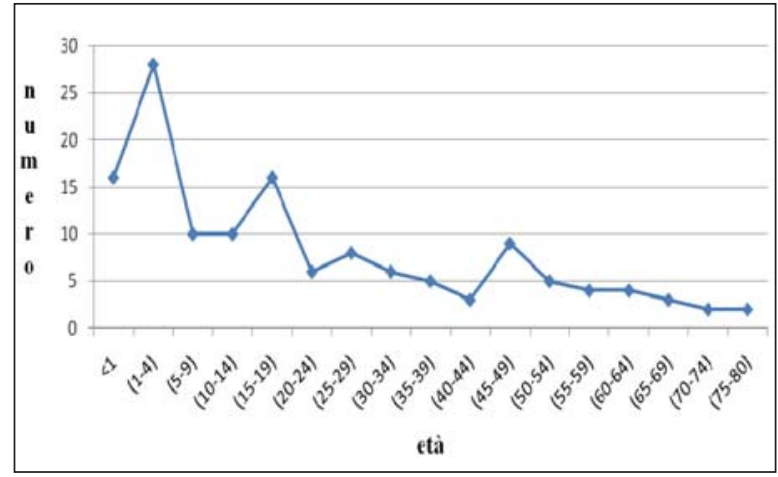

Figura I. Distribuzione delle classi di età di I 38 pazienti con meningite da Neisseria meningitidis.

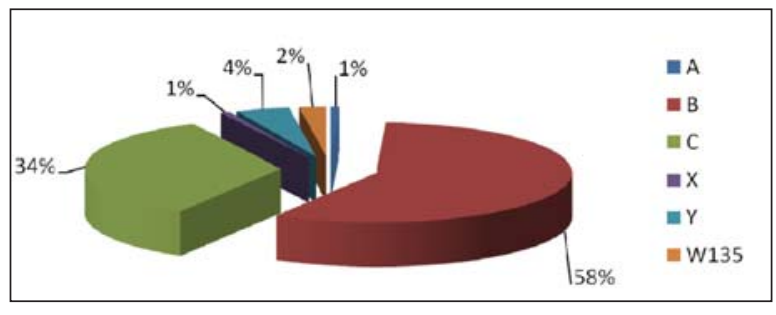

Figura II. Distribuzione del Sierogruppo di I 38 ceppi provenienti da altrettanti pazienti con meningite da Neisseria meningitidis.

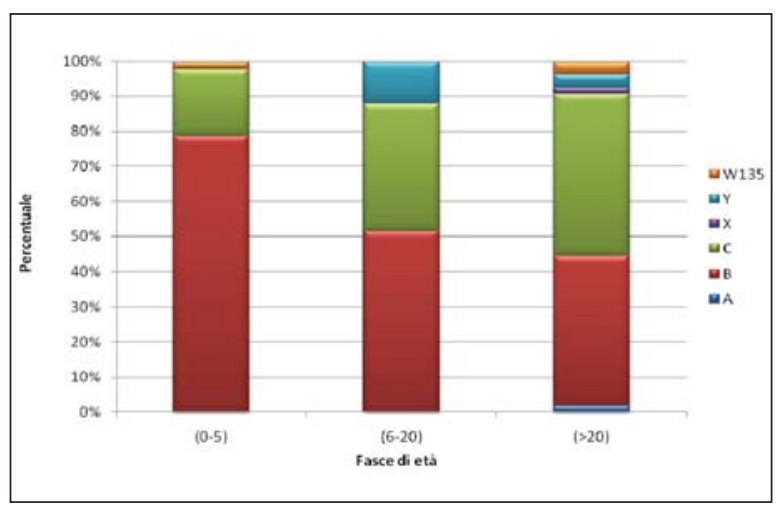

Figura III. Distribuzione del Sierogruppo di 138 ceppi provenienti da altrettanti pazienti con meningite da Neisseria meningitidis fasce di età.

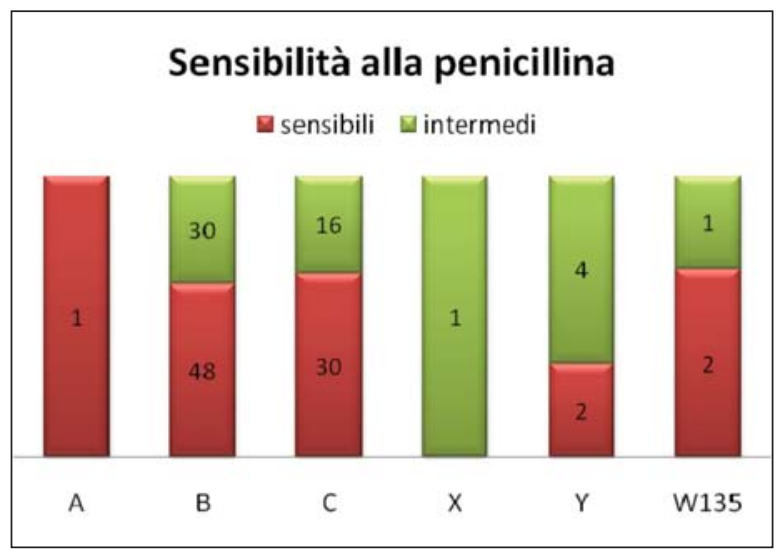

Figura IV. Sensibilità alla penicillina di 138 ceppi provenienti da altrettanti pazienti con meningite da Neisseria meningitidis. 
no essere considerati diversi; questo dato è supportato da dati epidemiologici e da segni distintivi visibili nei tracciati, indicando effettive differenze cromosomiche (Figura VII).

Il pattern 3 è costituito dai ceppi 8 e 9 che risultano molto diversi da tutti gli altri ceppi, ma indistinguibili tra loro (sierogruppo B) come è ben visibile nella Figura VIII.

\section{DISCUSSIONE}

Essendo il gruppo B il gruppo maggiormente rappresentato, conforta sapere che dal 2010 è in sviluppo un nuovo vaccino ricombinante meningococcico di gruppo B (5-7).

L'utilizzo di una tecnologia semplice e rapida nella risposta, quale il DiversiLab, dà la possibilità di individuare precocemente la presenza di cluster e di poter quindi adottare efficaci misure preventive.
I ceppi con diminuita sensibilità alla penicillina sono in evoluzione e bisogna monitorare attentamente la possibile comparsa di ceppi resistenti dovuti ad ulteriori modifiche del gene PenA. Nonostante la sensibilità alla rifampicina sia tutt'ora elevata è necessario valutarne sempre l'effettiva efficacia dato che è il principale farmaco utilizzato per la profilassi e acquisisce facilmente resistenze.

Dai risultati ottenuti mediante rep-PCR, si evince che i nove ceppi di $N$. meningitidis sono divisi in 3 cluster diversi fra loro.

Il primo cluster costituito da 4 ceppi isolati da 4 pazienti correlati fra di loro, di fenotipo $\mathrm{C}: 2 \mathrm{a}: \mathrm{P} 1.5$, il secondo costitutito da tre pazienti, anch'essi correlati fra di loro e appartenenti allo stesso fenotipo del cluster precedente, il terzo invece è costituito dal fenotipo B:15:P1.4. Questo sta ad indicare quanto il solo fenotipo non sia suf-

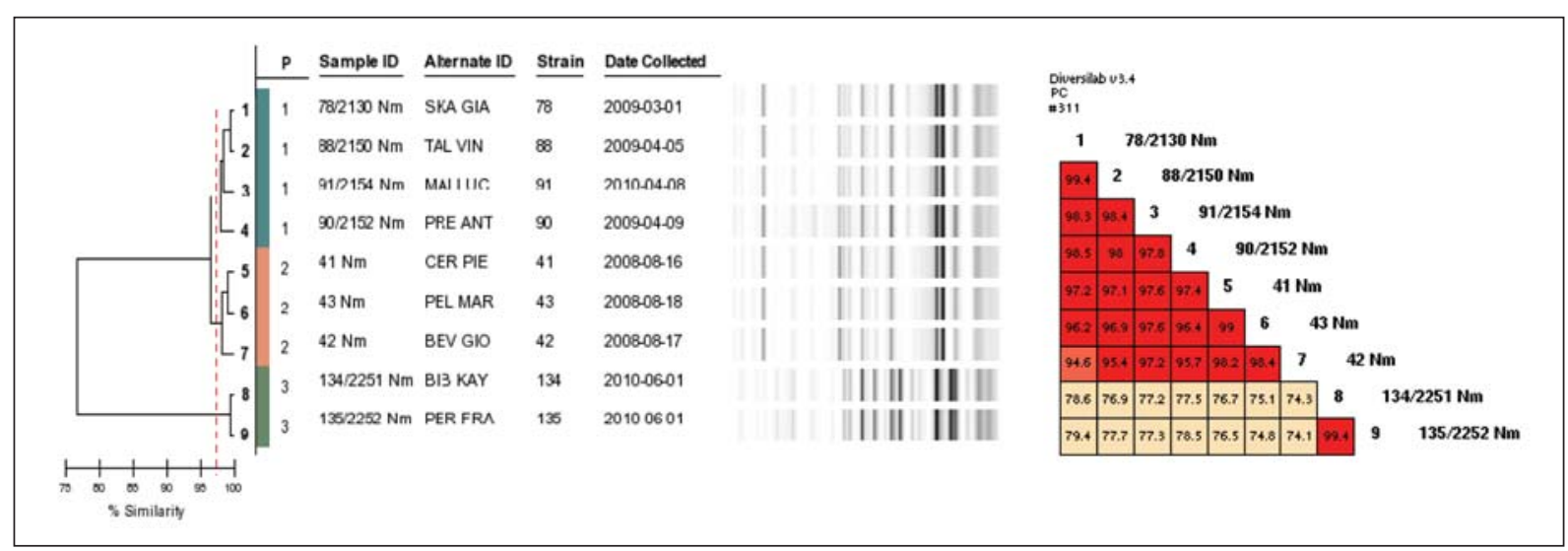

Figura V. Rappresentazione dei 9 ceppi testati con le rispettive similarità.
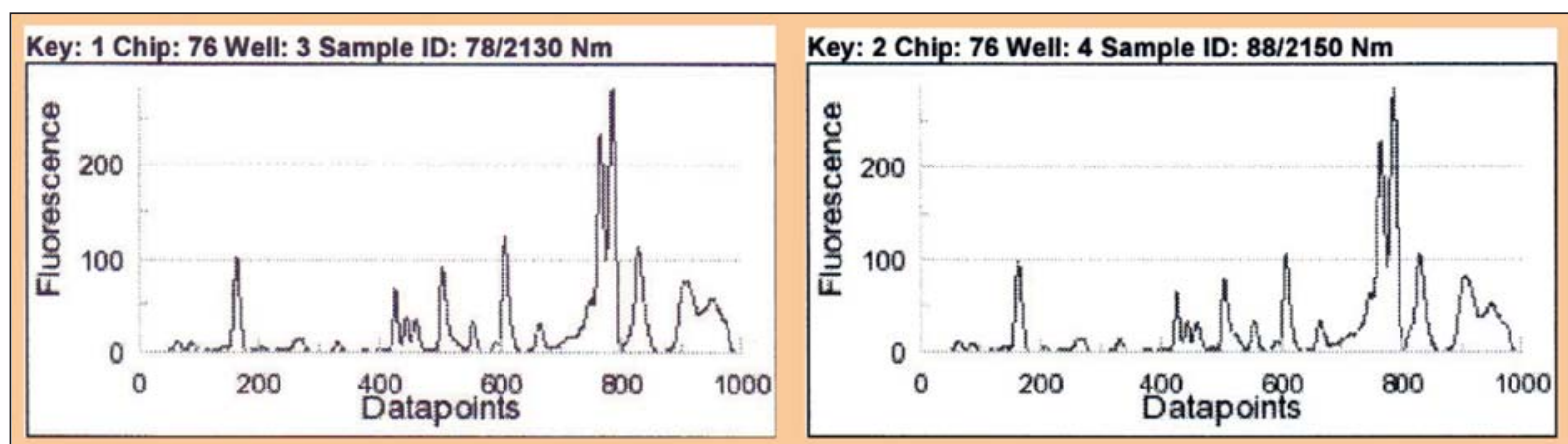

Key: 3 Chip: 76 Well: 6 Sample ID: 91/2154 Nm
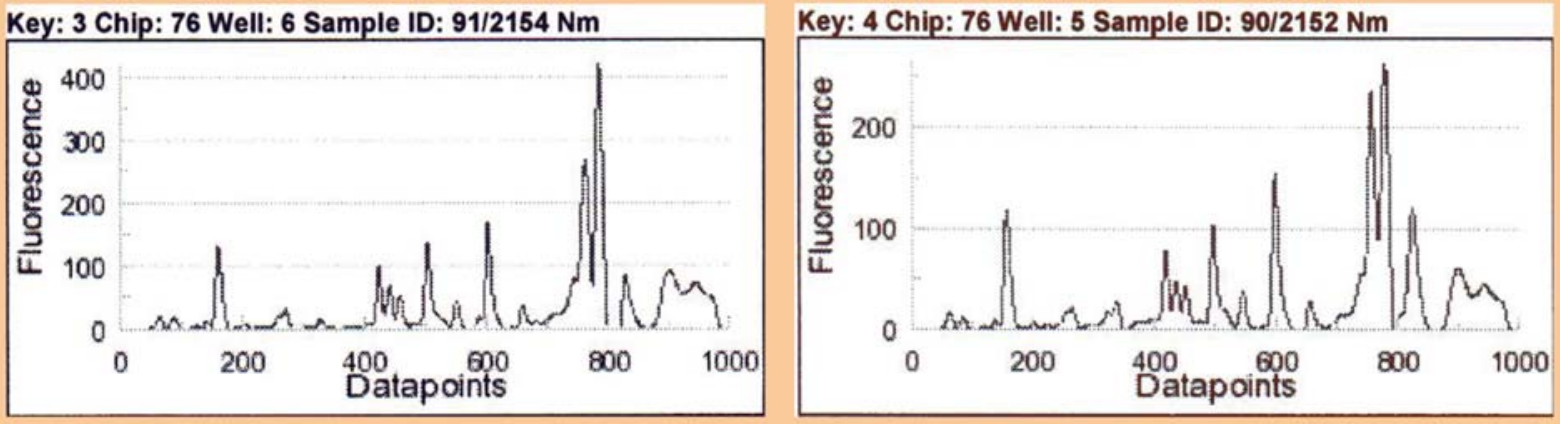

Figura VI. Pattern I: Elettroferogramma ceppi da I a 4. 
ficiente ad indicare la presenza di cluster epidemici, individuabili solamente mediante la genotipizzazione.

Un sistema di sorveglianza come quello attuato nella regione Lombardia e l'adesione degli operatori sanitari alle disposizioni vigenti rappresentano la base per una migliore valutazione epidemiologica della malattia meningococcica.
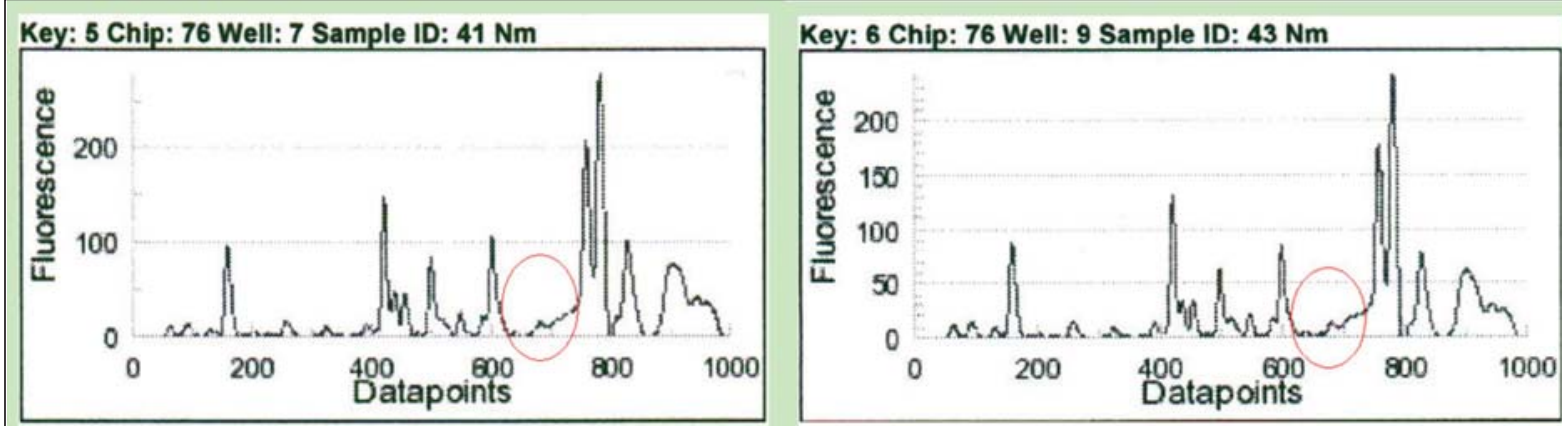

Key: 7 Chip: 76 Well: 8 Sample ID: $42 \mathrm{Nm}$

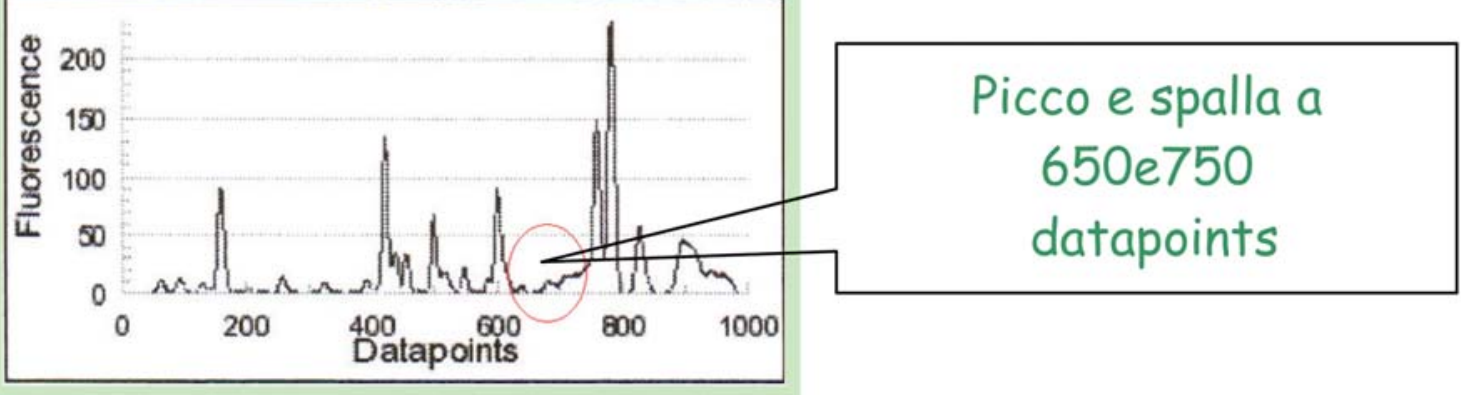

Figura VII. Pattern 2: Elettroferogramma ceppi da 5 a 7.

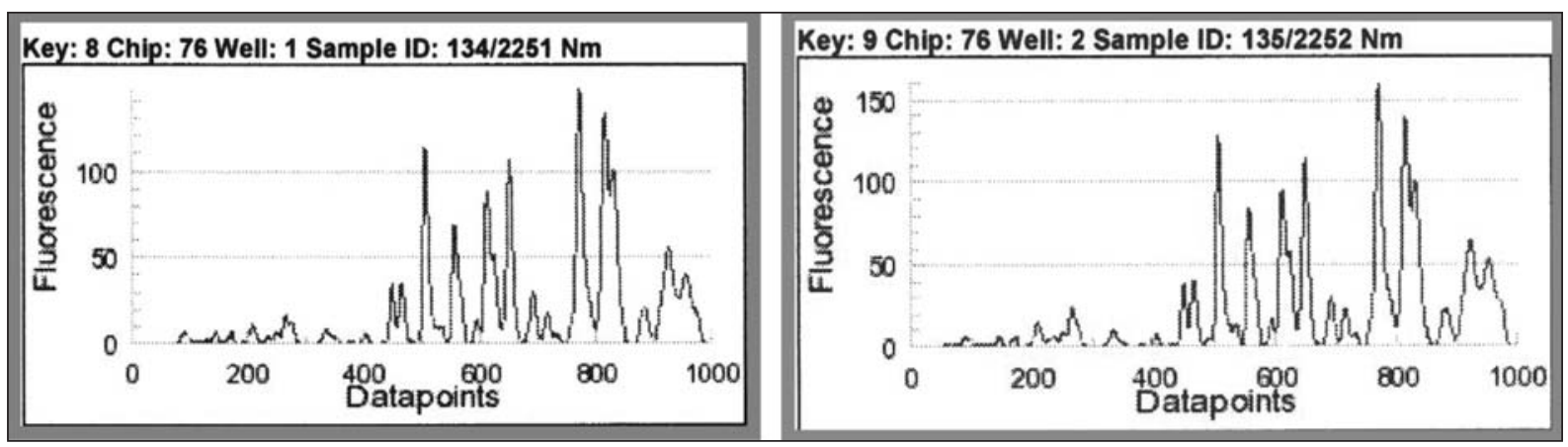

Figura VIII. Pattern 3: Elettroferogramma ceppi 8 e 9.

\section{BIBLIOGRAFIA}

1. Agrawal S, Nadel S. Acute bacterial meningitis in infants and Children: epidemiology and management. Paediatr. Drugs 2011; 13 (6): 385-400.

2. Bertrand S, Carion F, Wintjens R, Mathys V, Vanhoof R. Evolutionary changes in antimicrobial resistance of invasive Neisseria meningitidis isolates in Belgium during the period 2000-2010: Increasing prevalence of penicillin- non susceptibility. Antimicrob Agents Chemother. 2012; doi: 10.1128/AAC.06310-11. Article in Press

3. Biaukula VL, Tikoduadua L, Azzopardi K, et al. Meningitis in children in Fiji: etiology, epidemiology, and neurological sequele. Int J Infect Dis 2012; Article in Press.

4. Caugant DA, Tzanakaki G, Kriz P. Lessons from meningococcal cariage studies. Microbiol Rev 2007; 31 (1): 52-63.
5. Donnelly J, Medini D, Boccadifuoco G, et al. Qualitative and quantitative assessment of meningococcal antigens to evaluate the potential strain coverage of protein-based vaccines. Proc Natl Acad Sci U S A. 2010; 107 (45): 19490-5.

6. Giuliani MM, Adu-Bobie J, Comanducci M, et al. A universal vaccine for serogroup B meningococcus. Proc Natl Acad Sci U S A. 2006; 103 (29): 10834-9.

7. Gossger N, Snape MD, Yu LM, et al. Immunogenicity an Tolerability of Recombinant Serogroup B Meningococcal Vaccine Administered With or Without Routine Infant Vaccinations According to Different Immunization Schedules. JAMA. 2012; 307 (6): 573-82.

8. Katz LH, Zelazny A, Scharf, et al. Mass antibiotic treatment to stop an outbreak of meningococcal disease: a molecular analysis. Clin Microbiol Infect 2007; 13: 943-6.

9. Khatami A, Pollard AJ. The epidemiology of meningococcal disease and the impact of vaccines. Expert 
Rew vaccines 2010; 9 (3): 285-98.

10. Mastrantonio P, Sofia T, Neri A, Fazio C, Stefanelli P. Characterization of invasive meningococcal isolates from Italian children and adolescents. Clin Microbiol Infect 2007; 13: 100-3.

11. Neri A, Fazio C, Carannante A, Mastrantonio P, Stefanelli P. Molecular characterization of Neisseria meningitidis B:NT:P1.14/162 clonal complex respon- sible of invasive meningococcal disease in the north of Italy. Diagn Microbiol Infect Dis 2012; Doi: 10.10.16/j. diagmicrobio. 2012.01.003. Article in Press.

12. Tzanakaki G, Mastrantonio P. Aetiology of bacterial, meningitis and resistance to antibiotics of causative pathogens in Europe and in the Mediterranean region. Inter J Antimicrob Agents. 2007; 2: 621-9. 